\title{
Unmoderated Posters: Female Urology
}

\section{UP-62}

Removal of Sacral Neuromodulation Devices for MRI

Quirouet, Adrienne; Tenggardjaja, Chris; Vasavada, Sandip; Goldman, Howard; Moore, Courtenay; Rackley, Raymond

Cleveland Clinic, Cleveland, $\mathrm{OH}$, United States

Introduction and Objectives: Until recently, magnetic resonance imaging (MRI) has been contraindicated in patients with sacral neuromodulation devices. In February 2012, Medtronic released a guideline stating that 1.5Tesla MRI examinations of the head could be safely performed. However, it is not recommended that these patients undergo any other form of MRI. Thus, need for MRI represents one of the indications for removal of the device. To our knowledge, the rate of removal for this indication has not been published in the literature. The primary aim of this study is to evaluate how many patients undergo explant to have an MRI done and which of these patients subsequently get reimplantation.

Methods: We performed a retrospective chart review of all patients that underwent either revision or removal of a sacral neuromodulation device at a single institution from January 2011 to October 2013 by searching the current procedural terminology (CPT) code for this procedure. Clinic notes and operative reports were reviewed for relevant data.

Results: A total 75 patients underwent removal of a sacral neuromodulation device during this time period. 29 of these had lead removal only after and unsuccessful stage I procedure. Of the remaining 46 patients, 13 $(28 \%)$ underwent removal in order to undergo an MRI study. Only 3 of these patients reported efficacy of the device. None of the patients were reimplanted post MRI.

Conclusion: Need for MRI represents a significant proportion of sacral neuromodulation device removals. Reimplantation post-MRI was not observed.

\section{UP-63}

An Evaluation of Adoption and Adherence to Proposed Mesh Complication Terminology at Four Professional Society Meetings Quirouet, Adrienne; Tenggardjaja, Chris; Goldman, Howard

Cleveland Clinic, Cleveland, $\mathrm{OH}$, United States

Introduction and Objectives: In 2011, the International Urogynecological Association (IUGA) and International Continence Society (ICS) proposed newly defined terms to describe complications of prostheses and grafts in female pelvic floor surgery. Our objective is to evaluate adoption and adherence of this terminology.

Methods: Two reviewers (CT, AQ) independently read through all published abstracts presented at AUA, SUFU, ICS, and AUGS from 2010-2013. Abstracts were included if they dealt with incontinence or prolapse surgery involving prostheses or grafts. Male and animal/bench model testing abstracts were excluded. Discordant data was evaluated separately by a third reviewer (HG). Abstracts were then re-evaluated for the ICS/IUGA proposed terminology, specifically: erosion, contraction, prominence, separation, exposure, extrusion, compromise, perforation, dehiscence, sinus tract formation. Adherence was the use of proper ICS/IUGA terminology. Nonadherence was the use of "erosion". Hybrid adherence was the use of both. Results: 12042 abstracts were reviewed. 355 met inclusion criteria. Of these, 141 (39.7\%) were adherent, 140 (39.4\%) were non-adherent, 63 $(17.7 \%)$ were hybrid. Given the availability of the guideline since 2011, we evaluated 2012 as the cutoff for adoption. After publication of the guidelines, a significant decrease was seen in the abstracts utilizing the proposed terminology. (48.0\% vs. $38.3 \%, \mathrm{p}=0.006)$. However, a higher proportion of abstracts $(73 / 165,44.2 \%)$ demonstrated adherence to proper terminology after the publication of the guidelines compared to prior $(68 / 190,35.8 \%)$ but this was not statistically significant $(p=0.128)$.

Conclusions: A minority of abstracts dealing with female pelvic medicine prostheses and grafts adhere to the proposed terminology published by the ICS/IUGA. Although adherence seems to be improving, the value of proposed definitions and standards are questioned if they are not widely adopted. 